\title{
Precision thickness and refractive index imaging of molecular films
}

\author{
T. E. Balmer \\ Department of Materials, ETH Zurich, CH-8093 Zurich, Switzerland \\ M. Heuberger \\ Empa, Materials Science \& Technology, CH-9014 St. Gallen, Switzerland
}

(Received 5 May 2007; accepted 6 August 2007; published online 20 September 2007)

\begin{abstract}
We present an interferometric contrasting technique that allows visualizing the thickness and refractive index of a molecular film being part of an optical multilayer structure (etalon). Small wavelength shifts of the comblike etalon spectrum are transformed into measurable intensity variations using a second reference etalon (optical correlator), illuminated in series. A charge-coupled device camera acting as two-dimensional photometer is utilized to measure the optical spectral correlation (OSC) image. The performance of the here proposed optical spectral correlation method is demonstrated using very thin confined liquid films. We give a detailed signal-to-noise analysis. Subangstrom film thickness resolution is experimentally verified with single exposure images acquired at frame rates comparable to video standards $(\sim 25 \mathrm{~Hz})$. Finally, we describe the calibration procedures necessary to obtain an absolute quantification of the OSC image. () 2007 American Institute of Physics. [DOI: 10.1063/1.2777369]
\end{abstract}

\section{INTRODUCTION}

The thickness of thin films can be measured from analysis of the comblike spectrum of a Fabry-Pérot thin-film interferometer in a spectrograph or spectrometer, provided that the optical path $\Omega$ is a few times the wavelength $\lambda$. Working in transmission or in reflection is likewise possible. The wellestablished surface force apparatus technique is a well documented example where wavelength shifts of interference peaks are used to calculate the film thickness and the surface force. ${ }^{1}$ For optimal working conditions, a dielectric spacer layer is required to extend the total optical path. The thickness of a very thin film confined between two appropriate spacer layers can then be measured at high resolution-down to zero thickness.

Indeed, Fabry-Pérot interferometry is at the heart of several methods that were developed in the past, also to image the thickness of thin films. ${ }^{2}$ The simplest schemes use monochromatic light and work with so-called Newton rings, which are interference maxima along lines of constant optical path. Illumination with multiple colors has been envoked ${ }^{3}$ to improve the thickness sensitivity to about $\pm 1 \mathrm{~nm}$. The methods of highest resolution use broadband (white) light interferometry in conjunction with dispersive spectral analysis. However, the use of dispersive devices, such as spectrometers and spectrographs, entail severe limitations in terms of lateral resolution, speed, and cost. Here, we describe a simple film thickness imaging method that circumvents the above limitations by the use of a spectral correlator (second etalon) to replace the conventional dispersive spectral analysis.

\section{THEORY}

Let us consider a simple etalon consisting of a molecular film confined between two equally thick spacer layers terminated by metallic broadband reflectors (e.g., $40 \mathrm{~nm}$ silver), see also Fig. 1. When white light is directed normally through this simple etalon, partial reflections occur at all optical interfaces. The amplitude of the reflected light and the associated phase change $\phi$ are a function of the complex refractive index, $\mu=n+i k$, on both sides of an optical interface. In the etalon, multiple coherent beams of partially reflected light produce interference effects, depending on the wavelength as well as the thicknesses of the optical layers. Constructive interference occurs if the total phase difference $\delta$ between successive rays passing back and forth is $\delta$ $=2 \pi N$. The resulting comblike interference spectrum of an etalon consists of multiple intensity maxima that can be numerated by their chromatic order $N$. Variations of film thickness or film refractive index in the central molecular film result in wavelength shifts of these interference maxima-in analogy to a tunable resonator. If the thickness and refractive index of all other optical layers in the etalon are known, such wavelength shifts can be utilized to detect small changes of film thickness $D$ and refractive index $n$ in this central molecular film. The interferometric surface force apparatus (SFA) technique is based on this principle. ${ }^{4}$ The transmission spectrum of an etalon-including the peak wavelengthscan readily be predicted by theory. ${ }^{5,6}$ In practice, a mathematical inversion of this problem is necessary since the wavelengths of peak maxima are measured and the film thickness is to be calculated. ${ }^{1,7}$

\section{A. The principle of optical spectral correlation (OSC)}

When two etalons are illuminated in series by broadband (i.e., white) light, the transmitted intensity corresponds to the product of the two transmission functions $T_{1}(\lambda) T_{2}(\lambda)$. A maximum of light is transmitted when the etalon spectra are identical. Less light is transmitted if the comblike spectra are shifted relative to each other. In our case, the transmission 


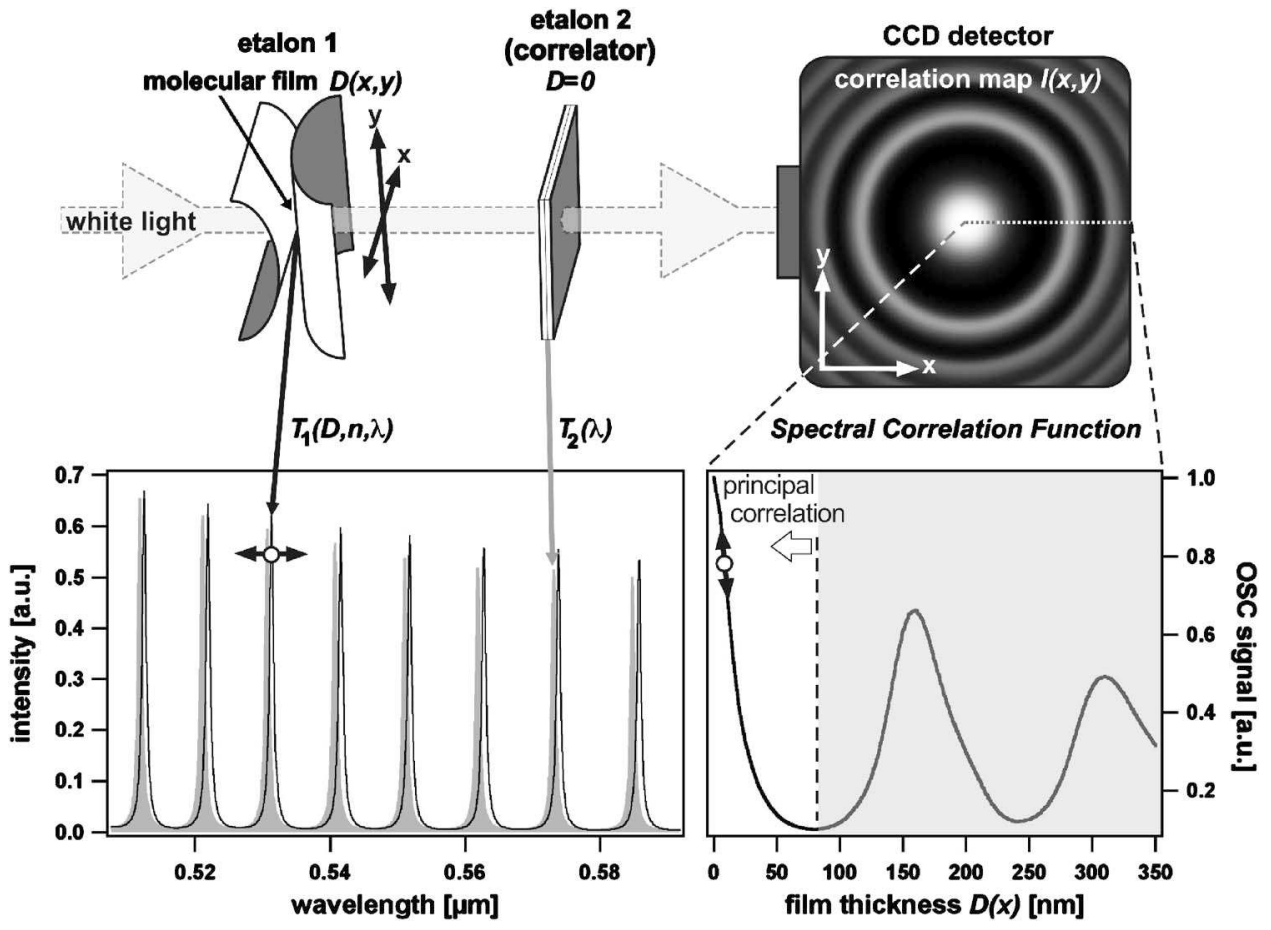

FIG. 1. The principle of optical spectral correlation (OSC). White light is directed through a first etalon confining a molecular film of variable thickness $D(x, y)$ and refractive index $n$. The transmitted light is analyzed by a second etalon (correlator) and detected with a CCD camera. The obtained image intensity is proportional to the spectral correlation function of the two transmission functions $T_{1}(D, n, \lambda)$ and $T_{2}(\lambda)$ of the two etalons, respectively. The resulting correlation map $I(x, y)$ yields a laterally resolved, real-time information about the confined medium, which then can be transformed into physical properties such as $D$ and $n$. A computed example is given for a typical etalon pair as used in this work (mica=4.3 $\mu \mathrm{m}$; Ag layers=40 nm; gap medium with $n=1.4$ ). The correlation function shows a strong decay in intensity for $D<50 \mathrm{~nm}$ (principal correlation). Within that range, the signal can also be used for calibration. At larger gap distances, weaker correlation maxima occur. The concentric ring structure seen in the correlation map is typical for the crossed-cylinder geometry of the interferometer used in the extended surface force apparatus (eSFA).

spectrum $T_{1}(D, n, \lambda)$ shifts due to changes of film thickness $D$ or refractive index $n$ of the central molecular film.

A gray level charge-coupled device (CCD) camera can detect a broad range of wavelengths. Therefore, the detected intensity is actually the spectral correlation function,

$$
I(D, n)=\int_{\lambda} C(\lambda) T_{1}(D, n, \lambda) T_{2}(\lambda) d \lambda,
$$

where $C(\lambda)$ accounts for the lamp spectrum and the CCD sensitivity.

As shown in Fig. 1, this spectral correlation signal is nonmonotonous and exhibits multiple maxima as a function of film thickness. The best sensitivity to film thickness changes is achieved near the principal correlation, i.e., film thickness $<50 \mathrm{~nm}$, and for a high finesse of the etalons. We also note the underlying analogy to the fast spectral correlation (FSC) maps introduced in an earlier reference. ${ }^{7}$

With its power for parallel processing, the OSC method is optimally suited for real-time imaging applications. For detection, the light, which is transmitted through the sample interferometer and the optical correlator in series, is focused onto the CCD camera. The measured gray level image directly corresponds to the spectral correlation map. As will be shown below, the characteristics of the principal correlation (e.g., slope and range) can be accustomed by the appropriate design of the optical correlator spectrum $T_{2}(\lambda)$.

\section{B. Design of the optical correlator}

The thin-film interferometer (etalon 1) typically used inside the extended surface force apparatus (eSFA) consists of two equally thick, backsilvered mica sheets mounted in a crossed-cylinder geometry, i.e., with a lateral distance $D(x, y)$ between the spacer layers; $x$ and $y$ denote the lateral dimensions of the imaging plane (cf. Fig. 1). A matching spectral correlator (etalon 2) would consist of a second set of identical mica sheets in contact. For this optical arrangement, the spectral correlation map $I(x, y)$ exhibits a concentric ring structure. The bottom part of Fig. 1 shows the calculated transmission functions (left) and the corresponding OSC function (right) of such a straightforward mica interferometer-correlator pair. As expected, the highest OSC (principal correlation) is obtained for zero film thicknesses. For simplicity, only the $D(x)$ cross section is shown here. The unambiguous operating range of the OSC measurement is limited to some $50 \mathrm{~nm}$ film thickness since the correlation function is not monotonous (multiple maxima). If we limit our discussion to the principal spectral correlation around $D=0$, we note that the intensity decays nonlinearly, and, depending on refractive index of the molecular film [cf. Fig. 2(c)]. It is the slope and shape of this intensity decay that determine the resolution and the dynamic range of the OSC measurement.

There are several ways to customize the transmission spectrum of the correlator etalon, to optimize for detection 

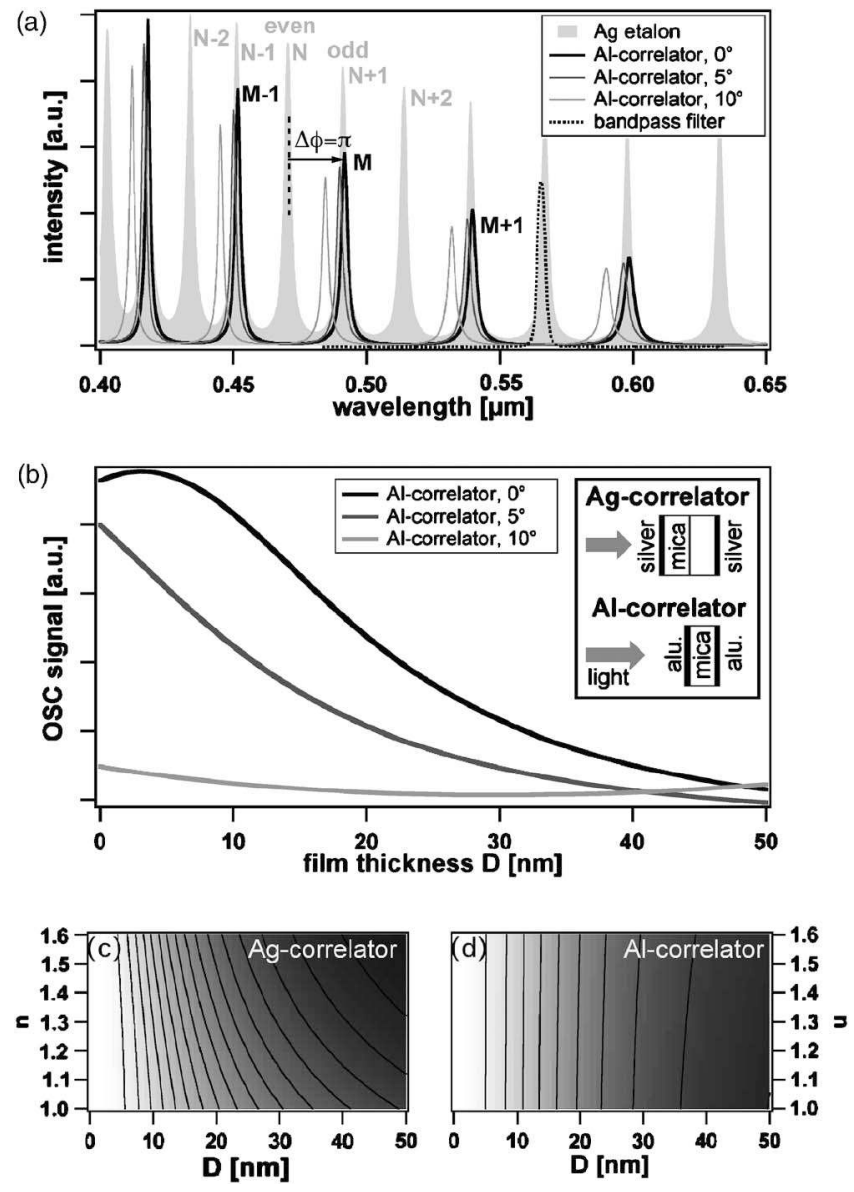

FIG. 2. Some design options for the optical correlator. (a) Calculated transmission function of an aluminum-coated correlator (Al correlator: mica $=1.7 \mu \mathrm{m}(1 \times)$; Al layer $=20 \mathrm{~nm}$, black curve) compared to a silver-coated etalon with twice the spacer thickness (Ag etalon: mica $=1.7 \mu \mathrm{m}(2 \times)$; $\mathrm{Ag}$ layer $=30 \mathrm{~nm}$, gray area). The reference maxima appear at the location of the odd-ordered fringes. A slight rotation of the correlator results in a fine shift of the peaks toward smaller wavelength (gray lines). Alternatively, a commercially available narrow bandpass filter can be used as a simplified optical correlator (dotted line). (b) Influence of the correlator-rotation on the shape of the OSC function. The region of highest sensitivity (i.e., highest slope) is shifted toward the smaller gap distance. The inset shows a schematic of the two mica correlator types. (c) Theoretical $D-n$ correlation map of an $\mathrm{Ag}$ etalon in series with a $\mathrm{Ag}$ correlator. The OSC function is sensitive to refractive index changes of the gap medium. (d) The $n$ sensitivity can be suppressed when using an $\mathrm{Al}$ correlator consisting of only one spacer layer.

range and sensitivity. These are the parameters that affect the shape and the position of the correlator's transmitted fringes.

The finesse $F$, i.e., sharpness of the comblike interference maxima, can be controlled by changing the reflectivity of the outer mirror surfaces. ${ }^{6}$ A higher mirror reflectivity results in sharper peaks with a high peak-to-valley ratio. The reflectivity can be adjusted with the thickness of the mirror coating or with the selection of the mirror material. The finesse also controls the slope and the range of the principal correlation. For example, one can increase the OSC detection range by choosing thinner $\mathrm{Ag}$ mirror coatings, albeit at the cost of a reduced film thickness resolution (i.e., less principal OSC slope).

Mathematically, the wavelengths of the constructive interference maxima depend on the total phase increment $\delta$ experienced by a ray of light traveling two ways through the interferometer. For the simplest correlator consisting of two spacer layers in direct contact, one has

$$
\delta_{2}=\frac{4 \pi}{\lambda} \mu 2 T \cos (\theta)+2 \phi,
$$

where $T$ is the thickness, $\mu$ the refractive index of the spacer, $\theta$ the angle of incidence, and $\Phi$ the phase change on reflection at the mirror coating.

We can adjust the peak wavelengths of the correlator etalon spectrum by changing any of the parameters in Eq. (2). For example, one can tune the offset of the spectral correlation function relative to the molecular film thickness.

We make use of the fact that the magnitude of the phase change is given by the mirror material and, for thin coatings, it strongly depends on the mirror thickness. ${ }^{6,8}$ It is thus readily possible to shift the wavelengths of the correlator etalon peaks by varying the mirror properties.

An interesting extension of this scheme is to design a correlator that contrasts the molecular film thickness $D$ and is insensitive to the refractive index $n$. Let us consider the spectrum of the previously described $\mathrm{Ag}$ etalon (i.e., two silvered mica sheets of equal thickness in direct contact). The transmission spectrum is shown as a light gray area in the background of Fig. 2(a). The total phase increment within this etalon is divided by 2 if it consists of only one silvered mica sheet. For such half-etalon correlator, we thus have $\delta_{2}$ $=\delta_{1} / 2$. In this special case, the correlator peaks selectively overlap with only the even-ordered interference maxima of etalon 1. Unfortunately, the correlator spectrum exhibits unfavorably low finesse in this case [i.e., broad peaks (not shown)] since it is only silvered on one side. In order to design a half-etalon correlator with a higher finesse, we propose to use aluminum mirrors instead on both sides of the mica sheet [cf. inset Fig. 2(b)]. Here, we can make use of the coincidence that the phase changes of $\mathrm{Al} / \mathrm{mica}$ and $\mathrm{Ag} / \mathrm{mica}$ interfaces obey the condition $2 \phi_{\mathrm{Al}} \approx \phi_{\mathrm{Ag}}+\pi$ and, therefore, we can realize a high-finesse half-etalon correlator with interference maxima near the wavelengths of the odd-ordered fringes of etalon 1 . We note that in the presented case, the correlator fringe of order $M$ overlaps with the interferometer fringe of odd order $N+1$, where $M=N / 2$, as illustrated in Fig. 2(a) with a plain black line. Since odd order fringes of etalon 1 exhibit negligible wavelength shifts with changes of refractive index in the molecular film, ${ }^{9}$ the OSC function is consequently independent of $n$ [cf. Fig. 2(d)].

Figure 2(b) illustrates that the optical spectral correlation function obtained with an $\mathrm{Al}$ correlator has a maximum for a molecular film thickness of $D \approx 5 \mathrm{~nm}$. As shown in Eq. (2), the phase increment in a thin-film interferometer depends on the cosine of the incident light angle $\theta$. We can now use this property to shift the Al-correlator peaks toward shorter wavelengths and hence fine tune the principal OSC maximum. It helps that this effect is small for angles below $2^{\circ}-3^{\circ}$. With correlator tilting, it is readily possible to adjust the region of maximal distance resolution to a desired film thickness range.

The preparation of customized correlators can be further extended but is often laborious. Therefore, the use of a simple interferometric bandpass filter as a correlator can be a 
cheap alternative for less demanding applications. Such interferometric filters are available for a range of different central wavelengths and bandwidths. With a set of filters available, one can choose an appropriate central wavelength. For interferometric bandpass filters, one can also use the aforementioned filter tilting for fine tuning of the OSC function. However, it is important to note that a custom-made OSC correlator will transmit considerably more light than a simple bandpass filter because all peaks of the comblike etalon spectrum are optically correlated. A high light intensity is an issue of central importance in the technical implementation of an OSC measurement since it limits the photometric signal-to-noise ratio and therefore the resolution.

\section{Quantitative photometry using a CCD camera}

Quantifying the OSC signal requires two steps: first, a quantitative photometric measurement by the $\mathrm{CCD}$, and second, a calibration to transform intensity into film thickness and/or refractive index.

The number of photoelectrons $I_{e^{-}}$that are generated when light falls onto a single pixel of a CCD detector can be expressed as

$$
I_{e^{-}}=\eta I_{h \nu}=\eta \frac{\Phi}{h \nu} A t_{\text {expt }},
$$

that is, the number of photons $I_{h v}$ times the quantum efficiency $\eta$ of the detector, whereas each pixel of area $A$ is exposed during the time $t_{\text {expt }}$ to the light intensity $\Phi$. Following exposure, the photoelectric charges are shifted to the readout register of the $\mathrm{CCD}$ chip and are then converted into digital A/D units, i.e., [ADUs] or [counts]

$$
I_{\mathrm{ADUs}}=G I_{e^{-}}=\frac{2^{\# \mathrm{bits}}}{f w c} I_{e^{-}},
$$

with $G$ the electronic gain of the A/D converter and $f w c$ the full well capacity of each single pixel.

The full well capacity is a measure of how many electrons can be created and temporarily stored on an individual CCD pixel before it saturates. Larger pixels have a higher $f w c$. It is convenient to normalize the intensity values by this saturation level,

$$
I=\frac{I_{\mathrm{ADUs}}}{2^{\text {\#bits }}}=\frac{I_{e^{-}}}{f w c} .
$$

The noise inherent to CCD exposure and signal processing effectively limits the resolution of the OSC method. Three sources of noise must be considered here, namely, shot noise, read noise, and dark noise. ${ }^{10}$ The shot noise or photon noise $\sigma_{P}$ is a result of the quantum nature of light. The number of generated photoelectrons obeys Poisson's statistics and is therefore given by the square root of the total number of electrons. Read noise $\sigma_{R}$ is mainly generated at the analogto-digital conversion and increases nonlinearly with readout speed. Faster data acquisition can thus only be performed at the cost of increased signal noise. High-end cameras offer the option to choose between different readout rates in a range of $1-10 \mathrm{MHz}$ typically. There is an additional source of noise even in the absence of illumination or readout ac-

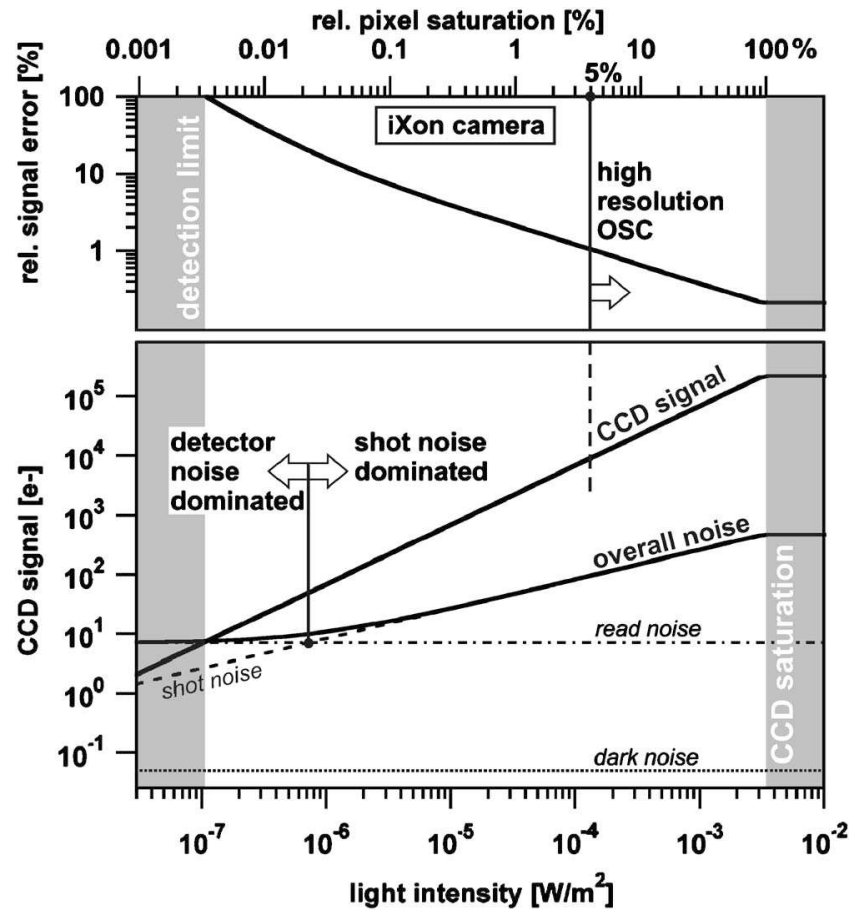

FIG. 3. Performance chart of a CCD detector. The three major sources of noise are shot noise, read noise, and dark noise (bottom). Above a certain light level, the shot noise becomes dominant and can be used to approximate the overall noise. Calculations are based on the OSC camera used in this work (iXon, Andor Technology). With increasing signal intensity, the relative error (noise/CCD signal) decreases until the pixels are saturated (top). High resolution OSC requires a relative error well below $1 \%$. In our case, this is achieved for pixel saturation above $5 \%$.

tion. It is the so-called dark current, which originates from thermal fluctuations in the semiconductor. The associated dark noise $\sigma_{D}$ depends nonlinearly on the temperature and linearly on the exposure time. It can be strongly reduced by cooling the detector. Highly sensitive cameras therefore operate with cooled CCDs, at temperatures as low as $-90{ }^{\circ} \mathrm{C}$.

An additional source of noise arises at the signal amplifying stage of the detector in the form of a microchannel plate (MCP) or a photomultiplier tube (PMT). This noise is inherent to the signal multiplying process and can be quantified by the so-called noise factor ( $n f)$, which also amplifies the shot noise. A new generation of CCDs is equipped with an on-chip electron multiplying (EM) device. Here, the charges are multiplied by impact ionization by passing through a biased gain register that is situated before the A/D conversion and signal amplifier. The result is an output signal for which the read noise becomes negligibly small. ${ }^{11,12}$ The noise factor of EMCCD based devices is typically 1.4, whereas for other amplification techniques, this quantity lies between 1.6 and 2.2. ${ }^{13}$

Combining the noise sources above, one can write an expression for the overall noise,

$$
\sigma_{\mathrm{CCD}}=\sqrt{\sigma_{R}^{2}+\sigma_{D}^{2}+\left(n f \sigma_{P}\right)^{2}}=\sqrt{\sigma_{R}^{2}+\sigma_{D}^{2}+\left(n f \sqrt{I_{e^{-}}}\right)^{2}},
$$

where $\sigma_{R}$ is the read noise, $\sigma_{D}$ the dark noise, $\sigma_{P}$ the shot noise, and $n f$ is the noise factor, which depends on the CCD amplification technology.

Figure 3 shows a CCD operation chart, where the CCD 
signal and the corresponding noise are plotted as a function of the incoming light intensity. The figure is representative of the specifications of the iXon CCD detector used in this work. Above a certain light level, the overall noise is dominated by the shot noise and the camera noise becomes negligible. At the highest intensities, we eventually reach the pixel saturation defined by the full well capacity. The relative signal error $\sigma_{\mathrm{CCD}} / I_{e^{-}}$(top graph) is plotted against the relative pixel saturation (top axis). It becomes clear that a low relative signal error can be achieved only when working close to the pixel saturation. Under these conditions, a sufficiently high input light level is necessary to reduce the exposure time and optimize for the speed of measurement. In conclusion, a camera with a large full well capacity (i.e., large pixel size) and high quantum efficiency is favorable. If we aim for a relative OSC signal error better than $1 \%$, we need a pixel saturation of at least $5 \%$. In this shot-noisedominated regime of operation, the OSC signal noise can be approximated by

$$
\begin{aligned}
& \sigma_{\mathrm{OSC}}\left[e^{-}\right]=n f \sqrt{I_{e^{-}},} \\
& \sigma_{\mathrm{OSC}}[\mathrm{rel}]=n f \sqrt{\frac{I}{f w c}},
\end{aligned}
$$

where $I_{e^{-}}$is the number of signal electrons and $I$ the relative intensity according to Eq. (5). The corresponding units are indicated within the brackets.

\section{The resolution limit of OSC}

The effective molecular film thickness resolution $\Delta D$ that one can achieve in practice is limited by the measured OSC signal variation $\Delta I$ and the slope of the OSC calibration function $|(\partial I / \partial D)|$. We have

$$
\frac{\Delta I}{\Delta D} \cong\left|\left(\frac{\partial I}{\partial D}\right)\right| \text {. }
$$

Using Eq. (7b) to estimate the signal variation, the filmthickness resolution of OSC can be written as

$$
\Delta D=\frac{n f}{|(\partial I / \partial D)|} \sqrt{\frac{I}{N_{S} f w c}} .
$$

Figure 4 shows this OSC film-thickness resolution for a typical experiment. The regime of highest resolution coincides with the highest slope, $|(\partial I / \partial D)|$, of the OSC calibration function where one can readily achieve angstrom resolution (gray area in Fig. 4, bottom graph). In our example, this is achieved in a film thickness range between 4 and $30 \mathrm{~nm}$. In Eq. (9), we also introduced the number of CCD images $N_{S}$ used for averaging (i.e., noise reduction). Averaging can be implemented both by accumulating a series of single exposure images or by taking the sum from a cluster of neighboring pixels (binning). This gain in film-thickness resolution is thus achieved either on the cost of time resolution or lateral resolution. In the example shown in Fig. 4, an averaging of $N_{S}=9 \times$ (i.e., $3 \times 3$ pixel binning) would be sufficient to achieve a subangstrom resolution over the full range of the principal correlation, $0-50 \mathrm{~nm}$.

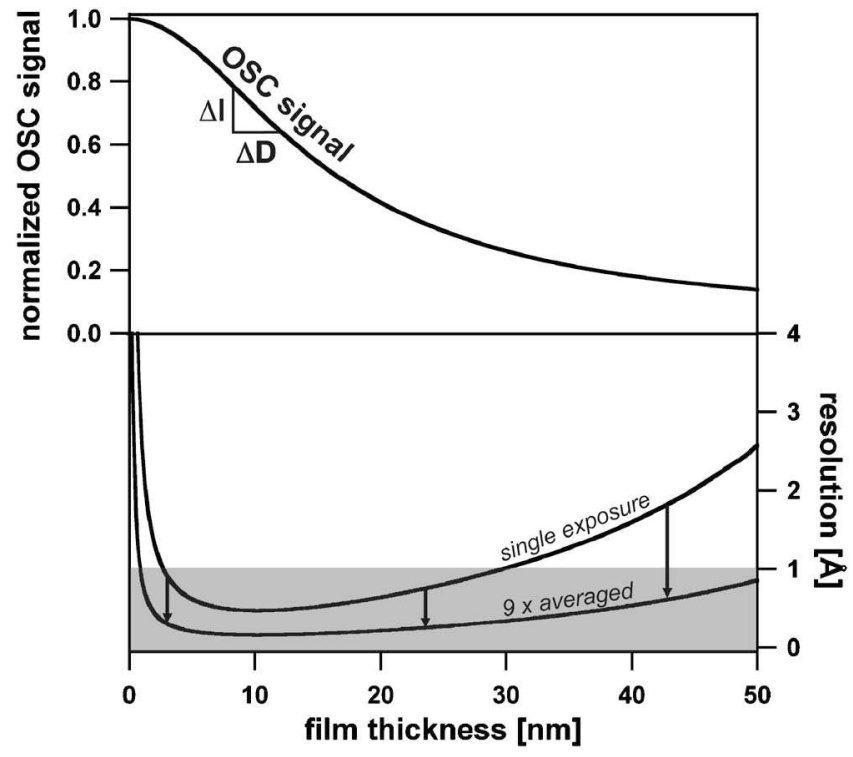

FIG. 4. OSC calibration function (top) and theoretical film thickness resolution (bottom). The correlation function was calculated for a basic interferometer and a custom-made Ag correlator (mica $=4.3 \mu \mathrm{m} ; \mathrm{Ag}$ layers $=40 \mathrm{~nm}$; gap medium with $n=1.4$ ). The resulting film thickness resolution was calculated according to Eq. (9) $\left(f w c=220000 e^{-} ; n f=1\right)$. Subangstrom resolution is readily achieved with single exposure images. For a $9 \times$ averaged signal, the regime of subangstrom resolution is extended to nearly the entire distance range from 0 to $50 \mathrm{~nm}$.

\section{EXPERIMENTAL SETUP}

The FSC and OSC measurements described in this work were obtained with the eSFA (Refs. 7 and 14) schematically shown in Fig. 5. White light from a $450 \mathrm{~W}$ Xe-arc lamp (Müller Elektronik Optik GMBH, Germany) is directed perpendicularly through etalon inside the fluid cell of the eSFA, from where it is focused on the pinhole aperture of a spectrograph with integrated CCD detector. We use a beam splitter (Edmund Optics Inc., USA) to simultaneously operate in the FSC (i.e., spectrograph) and OSC (i.e., CCD camera) modes. The direct beam enters the spectrograph (USB2000,

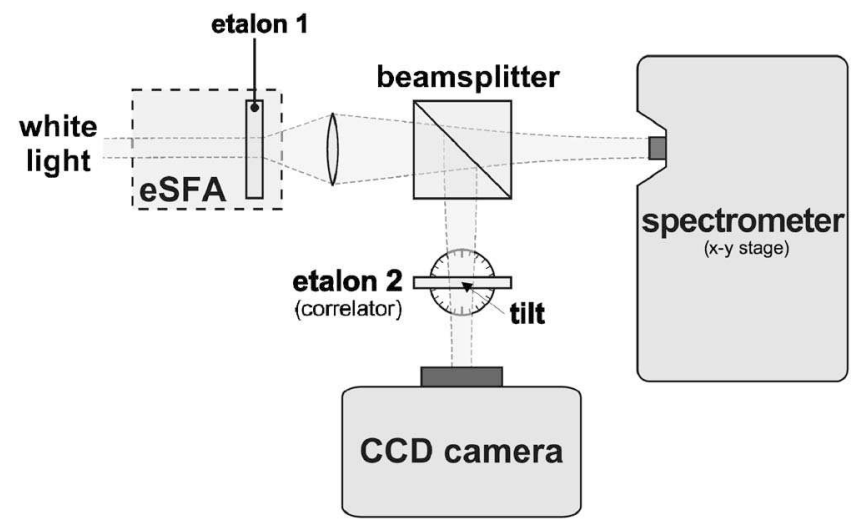

FIG. 5. Prototype OSC setup with dual detection capability. White light is directed perpendicular through etalon 1 inside the eSFA. A beam splitter is then used to simultaneously illuminate two different detection systems. First, the FSC detection using a spectrograph, and second, the OSC detection with a second etalon (correlator) and a CCD camera that provides a laterally resolved, real-time correlation image of the contact zone. Simultaneous operation of FSC and OSC is used for an absolute calibration of OSC image data. 
$385-715 \mathrm{~nm}$, Ocean Optics, USA) and is used for FSC measurement at one chosen point. ${ }^{7}$ The spectrometer is mounted on a motorized $x y$ stage (Thorlabs Inc., USA) for lateral scanning of this optical probe. Special care was taken to minimize stray light using a series of aperture stops.

The indirect beam is passed through the second etalon (correlator) before illuminating the CCD camera. The correlator is mounted on a rotational tilting stage (homebuilt, positioning accuracy of $\pm 0.5^{\circ}$ ) and additional $x^{\prime} y^{\prime}$-alignment capability. The right choice of the CCD camera is essential. It was done based on the considerations presented in Sec. II. We used an iXon DV887-BV from Andor Technology (Ireland). The CCD chip is a $512 \times 512$ pixel EMCCD sensor (CCD97-00, pixel size: $16 \times 16 \mu \mathrm{m}^{2}$; typical full well capacity: $220000 e^{-}$) produced by e2V-Technologies. The specified quantum efficiency at $575 \mathrm{~nm}$ is $92.5 \%$. The frame transfer (FT) design of the CCD removes the need of a shutter. This is a great advantage when working with a vibration-sensitive technique such as the eSFA. To reduce dark noise, the CCD chip is Peltier cooled to $-90{ }^{\circ} \mathrm{C}$. In addition, the CCD offers a choice of two output amplification modes, EM and the conventional mode. The camera is capable of 14 bit resolution at pixel readout rates of $10^{*}, 5^{*}, 3$, and $1 \mathrm{MHz}$ ( ${ }^{*}$ only in EM mode). This allows for a frame rate of up to $32 \mathrm{~Hz}$ for full frame transfer. Higher frame rates of $>100 \mathrm{~Hz}$ are possible with binning. According to the considerations above, the limiting parameter for speed is the exposure time required to realize a measurement in the shot-noise-dominated regime of operation. For the OSC data presented in this article, we typically operated the camera at $-50{ }^{\circ} \mathrm{C}$ in $3 \mathrm{MHz}$ conventional amplification mode or at $10 \mathrm{MHz}$ in EM mode (full frame) with exposure times ranging from 20 to $800 \mathrm{~ms}$.

The thin-film interferometer inside the eSFA was prepared from sheets of cleaved mica with a typical thickness between 1.6 and $4.4 \mu \mathrm{m}$, following the standard protocol for sample preparation used in our laboratory. ${ }^{15}$ For the OSC correlator, we cut one (Al correlator) or two (Ag correlator) additional pieces from the same mica sheet to assure identical thickness. All mirror coatings were thermally evaporated at a base pressure of $<4 \times 10^{-6}$ mbar. Ag correlators were prepared by bringing two backsilvered (typically $40 \mathrm{~nm} \mathrm{Ag}$ ) mica pieces into flat, direct contact. Al correlators were made from a single piece of mica and coated separately on both sides with typically $20 \mathrm{~nm} \mathrm{Al}$. The optical correlators were then fixed to a custom-made frame. A clean filter area of a few $\mathrm{mm}^{2}$ was sufficient for the OSC measurements. In some cases, an interference bandpass filter $[\lambda=532 \mathrm{~nm}$; full width at half maximum (FWHM) $3 \mathrm{~nm}$; Edmund Optics Inc., USA] was used as an optical correlator. Octamethylcyclotetrasiloxane (OMCTS) was used as a model liquid for the eSFA/OSC demonstration (Fluka, used as received).

\section{RESULTS}

In order to test the performance of OSC, we conducted a series of thin-film experiments with confined octamethylcyclotetrasiloxane (OMCTS). Spherical liquids are known to exhibit layering effects when confined to a few molecular diameters between atomically smooth mica surfaces. ${ }^{16}$ Due
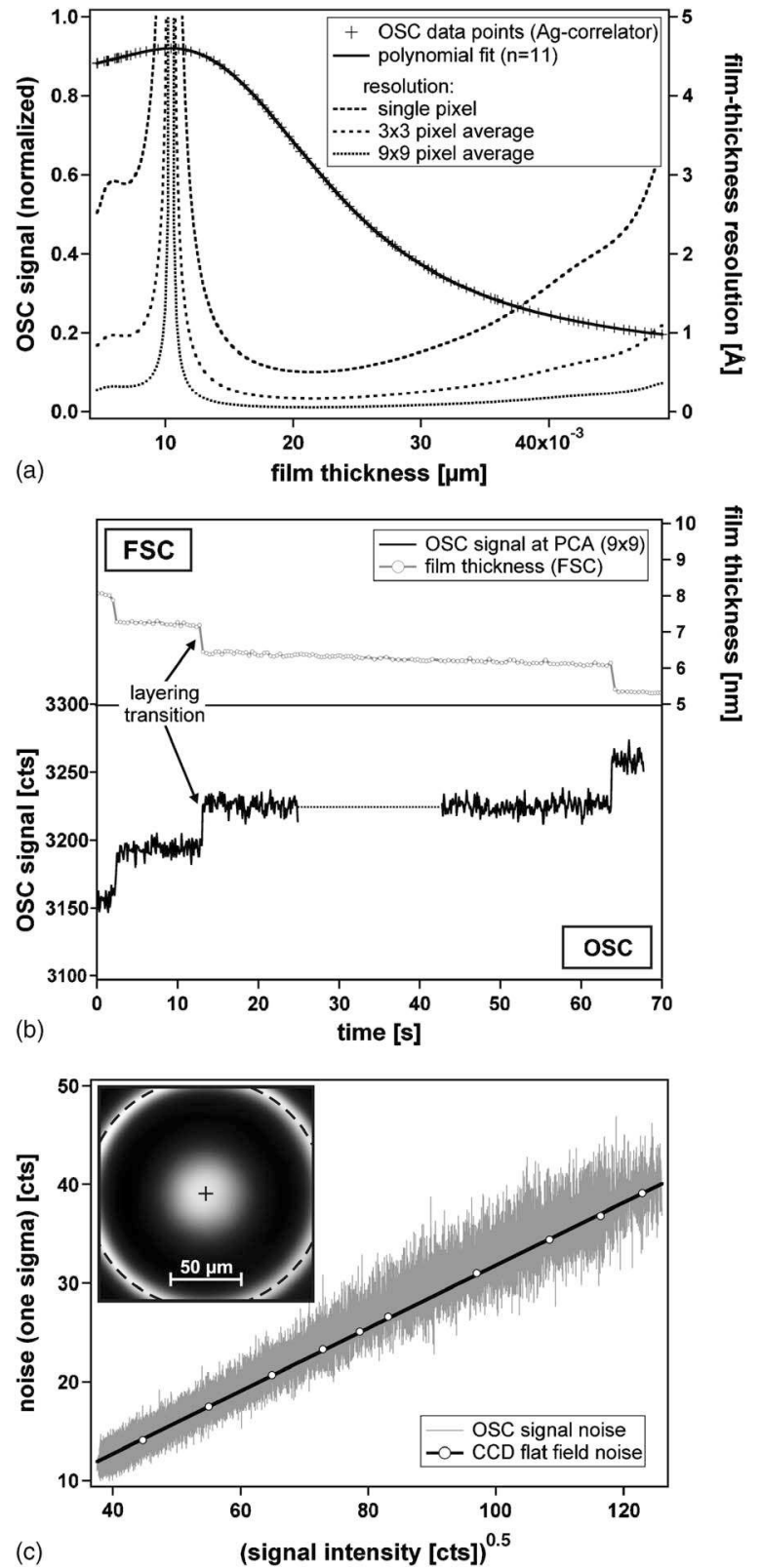

FIG. 6. Examples of measured OSC data. (a) Normalized correlation function acquired with an $\mathrm{Ag}$ correlator $($ mica $=4.37 \mu \mathrm{m})$ and the effective film thickness resolution calculated according Eq. (9) using $f w c=162000 e^{-}, n f$ $=1, N_{S}=1$ (dashed line), 9 (dotted), 81 (gray). (b) OSC signal at the PCA (Al correlator) in the regime of OMCTS layering transitions (bold black line). The signal is averaged over an area of $9 \times 9$ pixels, i.e., an effective sample area of $\sim 7 \times 7 \mu \mathrm{m}^{2}$ of the confined molecular film. After the described photometric corrections, the signal corresponds to the independently measured gap distance with FSC (white circles). (c) Characteristics of CCD signal noise plotted against the square root (expected shot noise) of the mean intensity level. The noise from flat-field measurements at intensity levels above 5\% saturation (white circles) suggests a purely shot-noisedominated behavior as verified by a linear fit (bold black line). The effective OSC signal noise follows the same trend (scattered gray line).

to the relatively large size of the molecule, the resulting oscillations in the free energy can be readily measured with the SFA. In a dry atmosphere, film-thickness transitions of step size 7-8 $\AA$ are commonly observed below a molecular film thickness of some $10 \mathrm{~nm}$. Figure 6(a) displays the OSC signal intensity measured with an $\mathrm{Ag}$ correlator, measured at the point of closest approach (PCA) between two curved mica surfaces. A series of 125 data points was recorded, while the 
mica surfaces were approached and the molecular film thickness $D$ was also independently measured using the absolute FSC method ${ }^{7}$ (reported on the $x$ axis). As expected from the OSC theory, we observe a strong increase of the correlation signal below a gap distance of $50 \mathrm{~nm}$ (cf. Figs. 1 and 4). The principal correlation peak is offset to $D \approx 10 \mathrm{~nm}$ because mica is birefringent and the two sheets were not optically aligned. It can be shown that a slightly misaligned mica in the Ag correlator can cause such shifts. Another source of smaller shifts is the adsorption of molecular films of contaminants onto mica surfaces in the correlator, which may be different in etalon 1 and etalon 2 due to the different medium in contact with the surface. Adsorbed water or airborne hydrocarbons are potential candidates for adsorbed molecular films. ${ }^{17}$ Such an offset does not impair the OSC measurement and can readily be corrected by the appropriate calibration. In the case presented in Fig. 6(a), we used a polynomial fit of 7 th to 13th degree to describe the OSC calibration function. This function was then stored as a fine-spaced look-up table and used to quickly transform the measured OSC signal into an absolute gap distance.

We also carried out OSC experiments with other types of correlators such as the $\mathrm{Al}$ correlator and the narrow bandpass filter. Furthermore, tilting of the correlator between $0^{\circ}$ and $12^{\circ}$ was routinely used to fine tune the effective range and region of highest resolution. For the study of structural forces in OMCTS, the range of interest lies between $0<D$ $<30 \mathrm{~nm}$, in particular, below $10 \mathrm{~nm}$ where molecularlayering transitions are typically observed. An important experimental difference between the three correlator types was found to be the total light level, which ultimately limits the system performance (i.e., resolution and speed). The highest OSC intensities could be realized with the Ag correlator, followed by the bandpass and the Al correlator (see also Fig. 8 below). Due to the higher absorption coefficient of aluminum, Al correlators (40 nm mirrors) transmit less than 1/1000 of the light transmitted by an Ag correlator with the same mirror thickness. Reducing the mirror thickness of the $\mathrm{Al}$ correlator allows for higher imaging rates $(\sim 1 \mathrm{~Hz})$, albeit at a reduced resolution $(\sim 5 \AA)$. Figure 6(b) displays the normalized OSC signal analyzed by an $\mathrm{Al}$ correlator in the regime of OMCTS layering transitions (black bold line). The steplike change in intensity coincides with the layering transitions observed in the independent FSC measurement [Fig. 6(a)]. A normalization of the OSC signal by the intensity of the light source was necessary to compensate for inherent small fluctuations of our Xe-arc light. To quantify the experimental resolution of our OSC setup, an in-depth experimental assessment of the CCD detector signal noise was done. From flat-field intensity measurements at different levels between $5 \%$ and $95 \%$ of saturation, we find the following empirical behavior for the camera's conventional amplifier mode (3 MHz readout),

$$
\sigma_{\mathrm{CCD}}[\mathrm{ADUs}]=0.318 \sqrt{I_{\mathrm{ADUs}}} .
$$

This power law is indeed expected for a shot-noisedominated mode of operation. Inserting Eq. (4) into Eq. (7) and comparing it with Eq. (10), we can inversely estimate the actual full well capacity of our system. We find $f w c$
$=162000$ electrons. This value agrees well with the nominal value specified by the manufacturer $(220000)$. In a second step, we acquired a series of OSC images with the interferometer surfaces in intimate contact. This series of $150 \mathrm{im}-$ ages was used to determine the mean and the standard deviation for each pixel. The analysis was done on the whole image area shown as inset in Fig. 6(c). The values span the full range of correlation intensities up to the maximum of the principal correlation. There is very good agreement between the noise behavior of the OSC signal and the independently measured flat-field noise of the CCD detector, which is purely shot noise dominated. The higher scattering of the OSC noise data points is due to the relatively lower number of samples (150 samples) as compared to the flat-field averaging (full frame: 262144 pixels). It is thus reasonable to use this shot-noise analysis as a basis to quantify the OSC resolution in terms of molecular film thickness.

To this end, we will use Eq. (9) and insert the measured full well capacity of the detector. Based on the measured OSC calibration function of the Ag correlator [Fig. 6(a)], we can indeed achieve subangstrom resolution for film thickness (one sigma) for each individual pixel of the CCD. The effective range of subangstrom resolution can be greatly extended if pixel averaging (e.g., binning) is used, as shown in Fig. 6(a). For example, with a $3 \times 3$ binning, we expect a molecular film thickness resolution well below $1 \AA$ over a range of $\sim 40 \mathrm{~nm}$ at a lateral resolution of $<3 \mu \mathrm{m}$.

The ultimate strength of OSC is to allow for a fast, precise, and accurate measurement of molecular film thickness in an entire region. Two examples of this remarkable capability are illustrated in Figs. 7(a) and 7(b). The top graph shows a quantified three-dimensional (3D) representation of the film thickness in the contact zone of the eSFA. A number of 3025 data points are shown spanning a lateral area of 42 $\times 42 \mu \mathrm{m}^{2}$. The OSC calibration function [cf. Figs. 4(a) and 6(a)] was used to transform intensity into film thickness. These data were acquired with an exposure time of $200 \mathrm{~ms}$, which allows a sample rate of $5 \mathrm{~Hz}$. This is a 36000 times gain of measurement speed compared to the same data acquired by the conventional scanning of the FSC probenotably, at no loss of resolution. Using OSC, it is now possible to follow the entire contact geometry during an eSFA experiment. Here, we have simply fitted a hemisphere into the two-dimensional (2D) data to get an effective local radius $R$ of curvature. Surface force measurements are commonly presented normalized to the local radius of curvature because this is a measure of the free energy at the interface (Derjaguin approximation). However, $R$ is not generally constant during the measurement. Figure 7(b) shows the evolution of $R$ during an isothermal compression of a thin fluid film of OMCTS. Two different curves are shown that correspond to a hemisphere fitted to a $31 \times 31$ and a $41 \times 41 \mu \mathrm{m}^{2}$ area, respectively. We observe a local flattening of the surfaces due to repulsive surface forces. The relative change of radius is more than $10 \%$ for the last $8 \mathrm{~nm}$ of the compressed liquid layer. As a control measurement, the $2 \mathrm{D}$ scan by conventional FSC [region of interest (ROI): $50 \times 50 \mu \mathrm{m}^{2}, 5 \times 5$ data points], taken prior to the isothermal compression at a gap distance of $340 \mathrm{~nm}$, resulted in a radius of $R=20.5 \mathrm{~mm}$. 


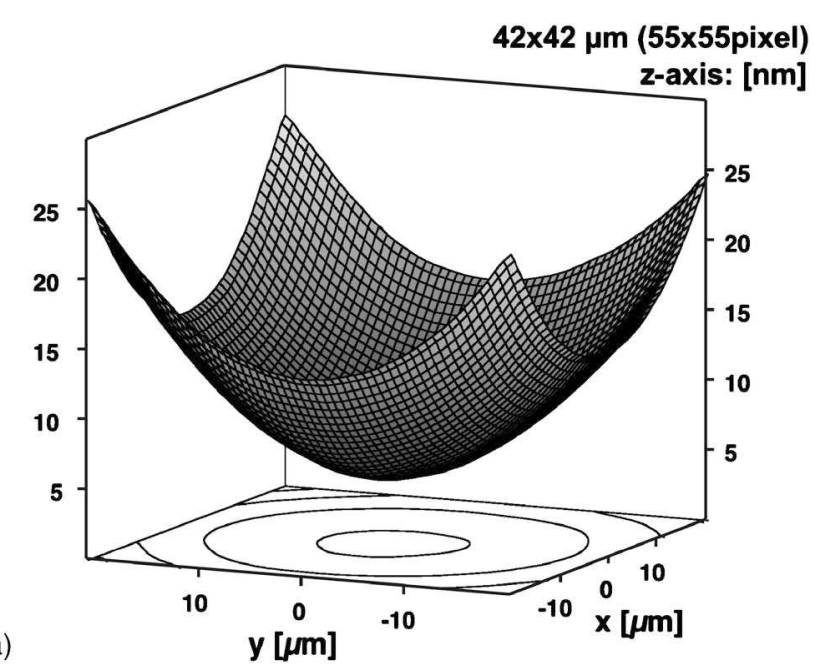

(a)

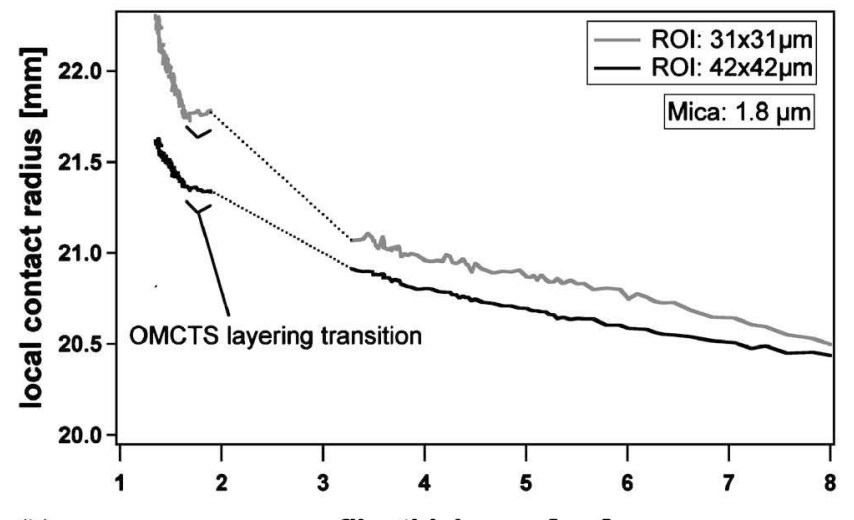

(b)

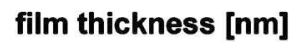

FIG. 7. Examples of calibrated OSC data. (a) 3D representation of the local contact geometry inside the eSFA. The data cover a region of 42 $\times 42 \mu \mathrm{m}^{2}$ and contains a total of 3025 data points around the point of closest approach (PCA). A correlation map was acquired during isothermal compression of a thin film of OMCTS (bandpass correlator, $200 \mathrm{~ms}$ exposure) and subsequently translated into film thickness using the appropriate OSC calibration function. (b) Evolution of the local contact radius as a function of the film thickness (at PCA). The radius is obtained by fitting a hemisphere to the 3D data points. The two lines result from fitting the radius in two regions of different sizes around the PCA, all acquired at $2 \mathrm{~Hz}$ during isothermal compression. The OSC measurement was paused between 2 and $3 \mathrm{~nm}$ (dashed lines). Below $2 \mathrm{~nm}$, an OMCTS layering transition occurred. The mica surfaces used in this experiment were $\sim 1.8 \mu \mathrm{m}$ thick and glued to silica disks of $R=20 \mathrm{~mm}$.

\section{DISCUSSION}

A very accurate photometric field measurement lies at the heart of the OSC method. This high degree of accuracy is possible, thanks to three measures, namely, the flat-field correction, the lamp normalization, and the OSC calibration (quantification).

\section{A. The flat-field correction}

Real OSC images are prone to image irregularities and imperfections due to the $10+$ optical layers the light has to pass. A closely related issue is homogeneous illumination by the lamp, which depends on geometry, reflections, or scattered light. Furthermore, the pixels of a CCD detector all have an individual photosensitivity and individual dark noise characteristics. While some of these effects can be mini- mized (e.g., aperture stops and CCD cooling), the high accuracy of the OSC measurements calls for a flat-field correction $^{11,18}$ of the following type:

$$
I_{i, j}=\frac{\left(S_{i, j}-\mathrm{DF}_{i, j}\right)}{\left(\mathrm{FF}_{i, j}-\mathrm{DF}_{i, j}\right)} \bar{M},
$$

where $I(i, j)$ is the corrected intensity image (pixel indices $i$, $j)$ as calculated based on the measured CCD signal $S(i, j)$. DF is the dark frame containing the detector background (dark current integral and A/D offset), FF the flat-field reference image, and $\bar{M}$ the background corrected average intensity of the flat-field image, $\bar{M}=\overline{(\mathrm{FF}-\mathrm{DF})}$. It is important that the exposure times of all images $S, \mathrm{DF}$, and FF is the same. In addition, the frames DF and FF are "noise-reduced" images obtained by averaging a large number of such exposures. The dark frame is readily acquired in total darkness. It is, however, more difficult to obtain a good flat-field image. While the optical correlator should be in place, optical correlations should not be measured. To reduce the OSC intensity modulations, one can separate the interferometer surfaces to a large gap distance of $>100 \mu \mathrm{m}$, where the correlation modulation is far from the principal correlation maximum and thus of smaller intensity. The drawback to this method is that at least one of the surfaces needs to be moved out of focus and therefore the resulting reference image not truly represents the same flat field as during the OSC measurement. A better alternative is to keep the surfaces closer than $\sim 1 \mu \mathrm{m}$ and approach the surfaces at constant speed (e.g., $5 \mathrm{~nm} / \mathrm{s}$ ) while accumulating a representative series of OSC images at different loci along the OSC function. If a suitable sequence of images is averaged, the intensity modulations induced by higher order correlations are averaged out and one obtains the wanted flat image FF. Typically, the sample surfaces should be moved over a range of $300-500 \mathrm{~nm}$ separation during this procedure.

\section{B. The lamp normalization}

A control experiment revealed that our Xe-arc lamp exhibits temporal intensity fluctuations of around 5\%, often irregular and over a time interval of several seconds. Presumably, these fluctuations stem from intrinsic arc instabilities. Since we aim for OSC measurements with a stability of better than $1 \%$ over an hour, we have to account for these effects. A simple solution is to measure the lamp intensity with an independent photosensor and mathematically normalize the measured OSC images. A more elegant way is to project a small fraction of the lamp light directly onto a dedicated section of the CCD detector. This can be realized, for example, by an optical fiber bypass or an optical aperture in the interferometer and correlator mirrors. The advantage of this second approach is an automatic synchronization with the OSC exposure time.

Here, we want to describe a third method in more detail that uses light outside the principal correlation, i.e., at film thickness of $>100 \mathrm{~nm}$. To get a reliable measure for the lamp intensity, we fitted an elliptical contour to the second maximum of the OSC function and averaged a fixed number of pixel values. For illustration of this procedure, the inset of 
Fig. 6(c) shows a dashed line over the second correlation maximum. Simultaneously, the center of this ellipse can be used to determine the location of the PCA. The data presented in Fig. 6(b) were normalized with this method. The normalized OSC signal is stable and perfectly reproduces the film thickness transitions independently measured using the FSC signal. The ellipsoidal contour fit of the second correlation has to be calculated for each image frame and is therefore best done in off-line analysis.

\section{The OSC calibration (quantification)}

Finally, the corrected and normalized photometric OSC intensity has to be transformed into film thickness. The possibility to carry out simultaneous OSC and FSC measurements is an advantage of our setup and is used here to establish an absolute, quantitative OSC calibration based on the absolute reference provided by FSC film thickness measurements. Notably, one can readily obtain an in situ calibration, which automatically accounts for all systematic photometric deviations between theory and the actual setup. To this end, we describe the steps necessary to determine the effective OSC function, which is subsequently used to translate OSC images into film-thickness maps.

The calibration is obtained by simultaneously measuring the film thickness (FSC) and the light intensity (OSC) for a range of different separations of the spacer layers in etalon 1. This can be done by varying the surface separation in the eSFA with the approach motor. As an alternative to such dynamic calibration, we propose a static calibration procedure for which the two surfaces are brought into contact. A noise-reduced OSC image is acquired simultaneously with a lateral FSC scan across the contact zone. Finally, the two data sets are plotted against each other to obtain the OSC calibration function. This method requires that the OSC detector is well aligned (i.e., rotation) and laterally calibrated in the $x y$ directions.

The OSC calibration has a limited lifetime, if, for example, the optical parts of the OSC setup undergo lateral drift. However, repeated calibrations obtained with an $\mathrm{Ag}$ correlator suggest a sufficient stability for at least $1 \mathrm{~h}$ of measurement. For extended experiments at subangstrom resolution, a periodic update of the calibration is useful.

\section{Dynamic measurements}

As mentioned in Sec. IV the main difference between the three correlator types used in this work (Ag correlator, $\mathrm{Al}$ correlator, and bandpass correlator) is the transmitted light intensity. This is of essential importance for experiments that require a high temporal resolution. In Fig. 8, we compare the normalized intensity level of the principal correlation for different etalon pairs. Depending on the correlator type, the light level varies over two orders of magnitude. By far, the highest OSC signal is obtained with $30 \mathrm{~nm}$ silver coatings in etalon 1 and etalon 2 . With that setup we achieve frame rates of $20-30 \mathrm{~Hz}$ with full frame images $(3 \mathrm{MHz}$ readout) and subangstrom film thickness resolution. For fast measurements, the sample rate can also be increased by making use of the binning ability of our CCD camera.

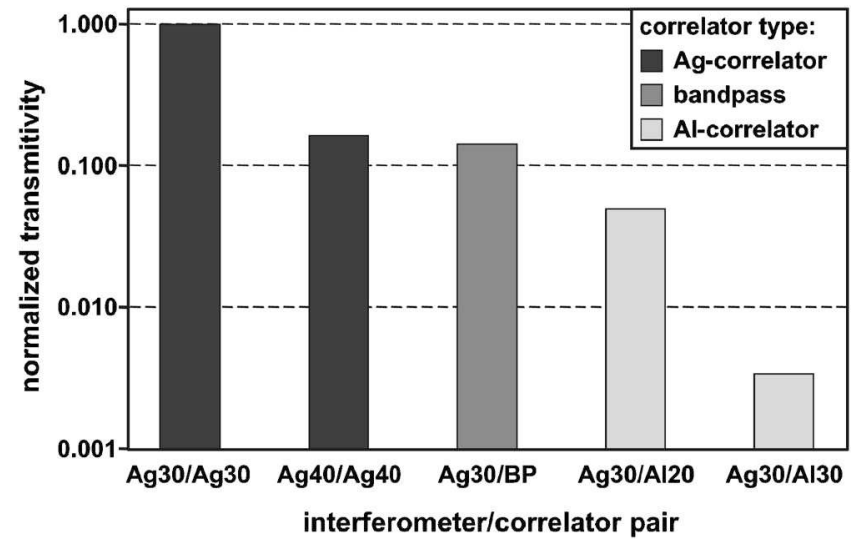

FIG. 8. Comparison of the normalized transmission function for different etalon/correlator pairs. Depending on the correlator type and the mirror coatings, the transmitted light level varies over two orders of magnitude. For fast measurements, i.e., high temporal resolution, a setup with high transmitivity is preferable. For example, one can achieve sample rates of $20-30 \mathrm{~Hz}$ without significant loss of resolution using $30 \mathrm{~nm}$ silver coatings for the etalon and correlator respectively $(\mathrm{Ag} 30 / \mathrm{Ag} 30)(\mathrm{Ag}=$ silver mirror; $\mathrm{Al}=$ aluminum mirror; $\mathrm{BP}=$ bandpass interference filter; the number indicates the corresponding thickness in $\mathrm{nm}$ ).

\section{E. Significance and future applications of OSC}

The high resolution achieved with OSC images does not depend on the resolving power of dispersive elements or on the exact wavelength calibration of spectrometers. The physical measurement is purely limited by photometric shot noise. This signifies that there is no physical limitation to further improvement of resolution, namely, by using a higher number of photons.

The OSC technique allows studying buried molecular films in many interesting situations. For example, it is possible to study the lateral repartition and evolution of molecular order in sliding contacts. This can yield valuable additional information for the understanding of structural details underlying molecular ordering in boundary lubricated model systems.

The application of OSC is, however, not limited to buried molecular films. Using the same principle, one can devise an imaging molecular adsorption sensor that can be operated inside or outside the eSFA. ${ }^{19}$ Indeed, OSC opens the pathways to a range of different imaging sensors that could be designed, for example, as attachment for conventional optical microscopy or as stand-alone devices. The high intensity modulation provided by the principal correlation even allows detection of changes in molecular films by low-cost industrial photosensors or even by the human eye, which allows for the development of hand-held devices for lower resolution applications. We note that sunlight is well suited to substitute the wide-band light source needed for OSC. This option can eliminate the need for electrical power altogether.

We have introduced and demonstrated the operation of a novel optical spectral correlation (OSC) scheme that can produce semiquantitative images of molecular film thickness and refractive index. Its real-time character and the subangstrom resolution allow for a number of novel experiments inside the extended surface force apparatus (eSFA). The OSC method is based on optical correlators that can be customized 
in a wide range. The quantitative photometric measurement of OSC images is optimal with a CCD camera in the shotnoise-dominated regime but can also be realized with lowcost industrial detectors or even the human eye, which opens a wide range of potential applications outside the eSFA.

\section{ACKNOWLEDGMENTS}

We would like to acknowledge N. D. Spencer for scientific discussions and support of the eSFA project. M. Elsener and J. Vanicek are thanked for excellent technical assistance. Furthermore, we would like to thank Hamamatsu for extensive CCD camera testing. This work was supported by the Swiss National Science Foundation.

${ }^{1}$ J. N. Israelachvili, J. Colloid Interface Sci. 44, 259 (1973).

${ }^{2}$ D. Platikanov, J. Phys. Chem. 68, 3619 (1964); A. D. Roberts and D. Tabor, Proc. R. Soc. London, Ser. A 325, 323 (1971); J. Radler and E. Sackmann, J. Phys. II 3, 727 (1993); F. Mugele et al., Colloids Surf., A 206, 105 (2002).

${ }^{3}$ G. J. Johnston, R. Wayte, and H. A. Spikes, Tribol. Trans. 34, 187 (1991). ${ }^{4}$ D. Tabor and R. H. Winterton, Proc. R. Soc. London, Ser. A 312, 435 (1969); J. N. Israelachvili and D. Tabor, ibid. 331, 19 (1972); J. N. Israelachvili and G. E. Adams, Nature (London) 262, 773 (1976); J. Chem.
Soc., Faraday Trans. 1 74, 975 (1978).

${ }^{5}$ S. Tolanksy, Multiple-Beam Interferometry of Surfaces and Films (Oxford University Press, London, 1949); M. T. Clarkson, J. Phys. D 22, 475 (1989).

${ }^{6}$ M. Born and E. Wolf, Principles of Optics, 6th ed. (Pergamon, Oxford, 1993).

${ }^{7}$ M. Heuberger, Rev. Sci. Instrum. 72, 1700 (2001).

${ }^{8}$ M. Heuberger, G. Luengo, and J. Israelachvili, Langmuir 13, 3839 (1997); W. H. Briscoe and R. G. Horn, J. Opt. A, Pure Appl. Opt. 6, 112 (2004).

${ }^{9}$ J. Israelachvili, J. Colloid Interface Sci. 44, 259 (1973).

${ }^{10} \mathrm{R}$. Berry, Choosing and Using a CCD Camera (Willmann-Bell, Richmond, 1992).

${ }^{11}$ C. G. Coates et al., J. Biomed. Opt. 9, 1244 (2004).

${ }^{12}$ See also http://www.emccd.com

${ }^{13}$ M. S. Robbins and B. J. Hadwen, IEEE Trans. Electron Devices 50, 1227 (2003).

${ }^{14}$ M. Heuberger, J. Vanicek, and M. Zach, Rev. Sci. Instrum. 72, 3556 (2001); M. Zach, J. Vanicek, and M. Heuberger, ibid. 74, 260 (2003).

${ }^{15}$ M. Zach, Ph.D. thesis, ETH Zurich, 2002.

${ }^{16}$ R. G. Horn and J. N. Israelachvili, J. Chem. Phys. 75, 1400 (1981); H. K. Christenson, ibid. 78, 6906 (1983); J. Klein and E. Kumacheva, ibid. 108, 6996 (1998).

${ }^{17}$ H. Poppa and A. G. Elliot, Surf. Sci. 24, 149 (1971).

${ }^{18} \mathrm{~J}$. C. Mullikin et al., in Image Acquisition and Scientific Imaging Systems, edited by H. C. Titus and A. Waks (SPIE, 1994), Vol. 2173, pp. 73.

${ }^{19}$ M. Heuberger and T. E. Balmer, J. Phys. D (to be published). 